\title{
Sleep apnoea syndrome: states of sleep and autonomic dysfunction
}

\author{
CHR IST I A G U I L L E I N A ULT, A R A T I L K I A N \\ K E N NETH LEHRMA N, L Y I A F O R O, A N D \\ W I L L I A M C. D E M E N T \\ From the Sleep Disorders Clinic and Laboratory, Departments of Cardiology and Pathology, \\ Stanford University School of Medicine, Stanford, California, USA
}

S UMMARY Eleven patients with upper airway apnoea during sleep (one with Shy-Drager syndrome) were monitored polygraphically for wakefulness, sleep, and cardiovascular variables. Systemic hypertension and most of the severe arrhythmias recorded during sleep were secondary to repetitive obstructive apnoea and were mediated through the autonomic nervous system. Sleep related elevations of pulmonary arterial pressure were not influenced by atropine or impaired autonomic functions. Upper airway sleep apnoea is sleep related; the type of sleep (REM or NREM) is critical in the appearance of abnormalities. The distinction between two patient subgroups (total sleep dependent and NREM sleep dependent) has haemodynamic, and possibly long-term, implications. Sleep apnoea syndrome should be looked for in patients with the Shy-Drager syndrome.

During the past five years considerable attention has been directed toward the sleep apnoea syndromes and the different types of apnoea observed during sleep (Guilleminault et al., 1976b). Speculation about the possible lethal role played by sleep apnoea in infants (Sudden Infant Death syndrome) (Robinson, 1974), and in adults (MacGregor et al., 1970) has led to systematic studies of sleep apnoeic patients. Haemodynamic changes occurring with upper airway apnoea during sleep (Coccagna et al., 1972; Tilkian et al., 1976), and the clinical symptoms associated with this syndrome in children (Guilleminault et al., 1976a) and adults (Guilleminault et al., 1977) have been outlined but very little is known about the underlying pathophysiology. Excessive daytime sleepiness has been demonstrated to be related to predominantly upper airway apnoea in sleep rather than to diaphragmatic paralysis (Guilleminault and Dement, 1977). The upper airway obstruction traditionally has been regarded as peripheral in aetiology, but Chekroverty et al. (1969) have sug-

\footnotetext{
Address for correspondence and reprint requests: Dr C. Guilleminault, Sleep Disorders Clinic and Laboratory, Stanford University School of Medicine, Stanford, California 94305, USA.

This research was supported by National Institute of Neurological Diseases and Stroke Grant No. NS 10727: Public Health Service Research Grant No. R.R.-70; Research Scientist Development A ward MH 05804 to Dr Dement, and INSERM to Dr Guilleminault. Accepted 18 January 1977
}

gested a central disturbance. This report outlines some factors favouring a central nervous system involvement in the upper airway/obstructive sleep apnoea syndrome.

\section{Patient population and clinical symptomatology}

From November 1974 to January 1976, 11 male patients, who gave informed consent to experiments (approved by the Stanford University School of Medicine Committee on the Use of Human Subjects in Research), participated in a specific study during wakefulness and sleep, conducted in collaboration with the Department of Cardiology.

The patients' ages ranged from 31 to 58 years (mean 44). All patients were referred to the Sleep Disorders Clinic for a complaint of excessive daytime sleepiness which had been present for two to 20 years before referral. Excessive daytime sleepiness was associated with hypnagogic hallucinations in 10 cases, but none presented with cataplexy or sleep paralysis. Eight patients had received dextroamphetamine or methylphenidate for several years without relief from the sleepiness. Ten patients (1-10) had no other neurological complaint. Patient 11 presented a Shy-Drager syndrome associated with an upper airway sleep apnoea syndrome and excessive daytime sleepiness. 
Patients 1-10 are presented as a single group, and their data are compared with those of patient 11 .

The average bodyweight of the 10 patients was $104 \mathrm{~kg}$ (range 78-143), and their average height was $182 \mathrm{~cm}$ (range 173 to 190). Eight patients were more than $10 \%$ overweight according to standard age-height-weight tables (Metropolitan Life Insurance Company, 1959). Two patients with borderline systemic hypertension were following weight-reduction programmes and were taking antihypertensive medication. Neurological evaluations, spirometric tests during wakefulness, and otolaryngological examinations were within normal limits in this study group.

Patient 11 was a 58 year old Caucasian male with a five year history of symptoms of gradual autonomic insufficiency. In 1969 the patient complained of progressive impotence, loss of sweating, and a decrease in thirst. Urinary incontinence, incomplete emptying of the bladder, and perianal numbness developed over the following three years. Before consultation at the Sleep Disorders Clinic he had noted increasing lightheadedness and dizziness when standing or exercising, and reported two episodes of orthostatic syncope. Two years after the onset of the first clinical symptoms the patient developed excessive daytime sleepiness. $\mathrm{He}$ complained of increasingly frequent daytime sleep spells that impaired his professional activities. At the time of hospitalisation, his weight was $85.4 \mathrm{~kg}$ and his height was $182 \mathrm{~cm}$. Cognitive function was normal, although frequent sleep spells accompanied by loud snoring were observed. Neurological examination revealed hoarseness and slurring of speech as well as slight extrapyramidal symptoms with a decrease in facial expression. Electroencephalogram, brain scan, and cerebrospinal fluid analysis were within normal limits. Otolaryngological examination revealed unilateral vocal cord paresis. The patient died during sleep, presumably from sleep apnoea, two weeks after the four day series of experiments. A complete necropsy with neuroanatomic study was performed.

\section{Experimental procedures}

Each patient's medication was interrupted for at least eight days before the study. Patients were admitted to the Clinical Research Center for four consecutive days. Spirometric and laryngological evaluations were performed on the first and third days of hospitalisation. Each nocturnal sleep period was monitored polygraphically for sleep and respiration using a standardised protocol previously described (Guilleminault et al., 1975b).
On the fourth day each patient underwent right heart catheterisation. Swan-Ganz thermodilution catheters were positioned in the right pulmonary artery, and a polyethylene catheter was introduced into a femoral artery. The intravascular catheters were kept open by a continuous flush system, and patients were left undisturbed for 10-12 hours after 2200. Pulmonary arterial pressure (PAP) and systemic arterial pressure were continuously monitored on a Honeywell strip chart recorder. Arterial pressures and ECG were recorded on a Sony tape recorder.

For 12 hours on the second night and for 24 hours that included the haemodynamic monitoring, the ECG was monitored continuously in each patient using a portable single channel Avionics recorder. All ECG tapes were processed by computer methods described previously (Tilkian et al., 1976). One patient (case 7) also received an intravenous injection of atropine sulphate $(1 \mathrm{mg})$ during an early morning period of rapid eye movement (REM) sleep at the end of his haemodynamic study (day 4).

Several months after the study, five patients (cases 2, 3, 4, 5, and 9) underwent two successive all-night recordings of sleep and respiration, including a 24 hour ambulatory ECG recording. Oral atropine sulphate $(1.2 \mathrm{mg})$ was administered just before the onset of sleep and again at 0200 .

Patient 11 underwent an identical four day programme. On the fourth day, right heart catheterisation was followed by specific interventions and drug trials [control Valsalva manoeuvre, cold pressor test, mental stress, amylnitrite inhalation test, tilt table test $-30^{\circ}, 45^{\circ}$, $60^{\circ}$, norepinephrine (12 mg i.v.), angiotensin 1.0 $\mathrm{mg}$, metaraminol $0.65 \mathrm{mg} /$ minute, atropine 1.0 $\mathrm{mg}$ i.v.] to evaluate the impact of the autonomic nervous system lesion upon cardiovascular variables. At the end of this evaluation, catheters were kept open by the continuous flush system, and overnight haemodynamic studies were performed.

\section{Results}

\section{SLEEP}

All patients had abnormal sleep patterns. Sleep stages were difficult to identify. As previously reported in another group of sleep apnoeic patients (Guilleminault et al., 1975a), nonrapid eye movement stages (NREM) of sleep resembled those seen in normal subjects: the repeated apnoeic episodes were often associated with low amplitude, mixed frequency waves in the EEG, and sudden bursts of high amplitude theta or delta 
waves resembling stage two NREM sleep.

In patients 1-10 the average total sleep time was 358 minutes with the following average proportions of sleep stages: stage 1 NREM sleep was $39.7 \%$, stage 2 NREM sleep $44.1 \%$, stages 3 and 4 NREM sleep $0.8 \%$, and REM sleep $15.4 \%$ of the total sleep time (Table).

Table Mean percentage of total sleep time spent in NREM sleep

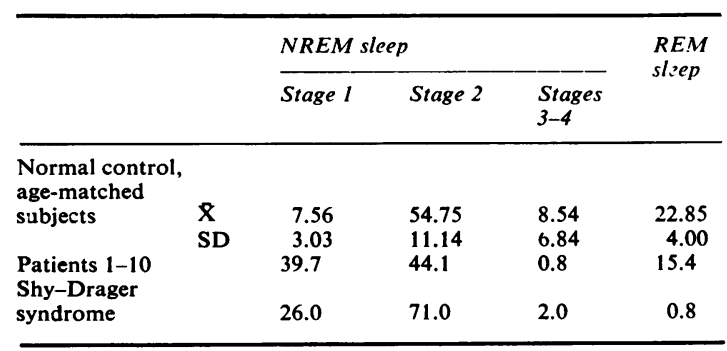

Patient 11 had similarly disturbed NREM sleep. Average total sleep time was 260 minutes, with the following sleep stage percentages: stage 1 NREM sleep was $26 \%$, stage $271 \%$, stages $3-42 \%$, and REM sleep $0.8 \%$ of total sleep time. Thus, REM sleep was greatly reduced in this patient as compared to normal levels as well as in the other patients.

\section{RESPIRATION DURING SLEEP}

During sleep, all patients had repeated interru:tions of air exchange (apnoeas) that lasted 10-90 seconds. When respiration was monitored around the clock, no apnoeas were seen during wakefulness; apnoeic episodes occurred only during sleep in all patients.

In patients 1-10 the average number of apnoeas during nocturnal sleep was 411 (range 195-543), which accounted for an average of 211 minutes (range $=71-322$ minutes) or $58 \%$ (range $26 \%$ to $69 \%$ ) of total nocturnal sleep.

Patient 11 was not different from the above group. He had an average of 452 apnoeas during nocturnal sleep, accounting for an average of 143 minutes $(55 \%$ of total sleep time) without air exchange during nocturnal sleep.

The three types of apnoea (central, mixed, and obstructive) that have been described in the literature (Tassinari et al., 1972) were seen in each patient on any given night. However, $90.9 \%$ of the air exchange pauses during sleep were related to obstructive sleep apnoea; $3.35 \%$ and $5.75 \%$ were central and mixed respectively. (Case 11: $91 \%, 4 \%$, and $5 \%$ respectively). These results were in accordance with previous reports of other sleep apnoeic patients (Sackner et al., 1974; Guilleminault et al., 1975a; 1975b; Tilkian et al., 1976). When the temporal distribution of the apnoeic episodes during sleep was analysed, patients could be subdivided into two groups. Group A (cases 2 and 6) experienced apnoeic episodes only during NREM sleep; their respiration was entirely normal during REM sleep. Group B (all other cases) had apnoeic episodes during both NREM and REM sleep. Case 11 -with a very small proportion of
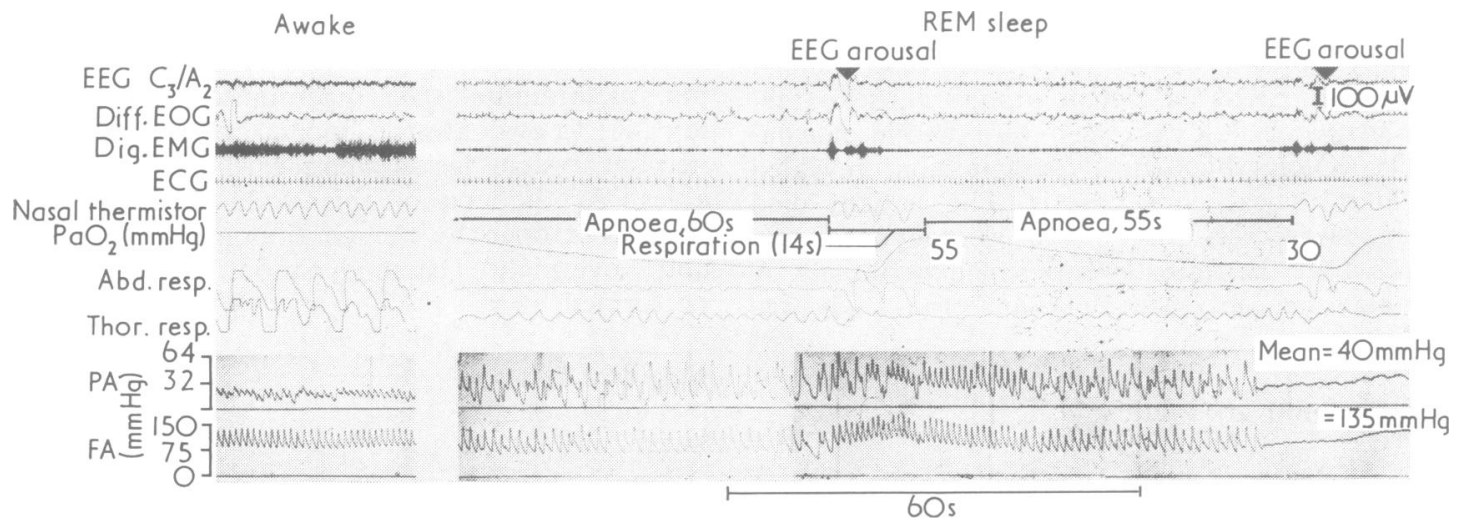

Fig. 1 Awake and sleeping polygraphic recording in patient 8 . Wakefulness is characterised by regular sinus rhythm and rhythmic excursion of abdominal and thoracic strain gauges; air flows through the nostril (nasal thermistor). Oxygen saturation is $92 \mathrm{mmHg}$. During REM sleep period the patient had repetitive obstructive apnoea and $\mathrm{PaO}_{2}$ oscillated between 55 and $30 \mathrm{mmHg}$. Changes in pulmonary and femoral arterial pressures are shown. Bradycardia during the apnoea is noticeable. Arrhythmia can be noted just before resumption of respiration at the end of the first apnoea.

Diff.EOG = differential electro-oculogram; Dig.EMG =digastric electromyogram; Abd.resp=abdominal respiration; Thor.resp $=$ thoracic respiration; $P A=$ pulmonary arterial pressure; $F A=$ femoral arterial pressure. 
REM sleep-was part of the latter group. Oximetric curve and blood gases measurements, which indicated that all patients developed alveolar hypoventilation during sleep with moderate to severe hypoxaemia and slight to moderate hypercapnia (Tilkian et al., 1976), confirmed the normality of respiration during each REM sleep period in Group $\mathrm{A}$. $\mathrm{PaO}_{2}$ and $\mathrm{PaCO}_{2}$ were normal during REM sleep in contrast to values recorded in Group $\mathrm{B}$ patients whose REM sleep $\mathrm{PaO}_{2}$ measurements ranged between 25 and $55 \mathrm{mmHg}$.

\section{HAEMODYNAMIC INVESTIGATION}

During sleep, cases 1-10 all showed increases in pulmonary arterial pressure (PAP). There was a progressive increase with each apnoeic period; pressures returned towards control levels at resumption of respiration. This led to a progressive rise in PAP from onset of sleep until wakefulness, independent of sleep stage or sleep state, in Group B. Cases 2 and 6 (Group A) presented a different pattern: PAP values rose progressively during NREM sleep but returned to completely normal values during REM sleep periods when respiration was normal. Pulmonary arterial pressure never reached the elevated value seen in other patients at the end of the total nocturnal sleep period, and pulmonary hypertension, when it occurred, was moderate. Similarly, systemic arterial pressure increased progressively in association with repetitive apnoea during sleep in eight patients. Cases 2 and 6 (Group A) showed this increase only during NREM sleep; each time a REM sleep period occurred, systemic pressure returned to normal. The cyclic reappearance of REM sleep periods during the night accounts for the minimal total rise in systemic pressure observed in these two patients.

In case 11 , during wakefulness, there were grossly abnormal responses to all intravenous drug tests as previously reported in the ShyDrager syndrome, indicating severe autonomic dysfunction of central and peripheral origin (Bannister et al., 1967). The response to intravenous atropine sulphate indicated some residual parasympathetic innervation of the heart. During sleep, in contrast to the other sleep apnoeic patients, case 11 had a slight decrease in systemic blood pressures and moderate pulmonary hypertension.

\section{ARRHYTHMIAS AND SLEEP}

During sleep, marked sinus arrhythmias were seen in cases 1-10. These arrhythmias were characterised by progressive sinus bradycardia levels of 25 beats per minute during apnoea and sinus tachycardia up to 120 beats per minute at resumption of respiration. More severe arrhythmias were observed in some patients. Apnoea and sinus bradycardia were accompanied by ventricular premature contraction in six patients, asystole in two patients (for 3.5 and 6.5 seconds) (see Fig. 3), second degree heart block in two patients, and ventricular tachycardia in one patient. None of these marked sinus arrhythmias or more severe ones were seen during REM sleep in cases 2 and 6 (Group A). Case 11 presented phasic minute-by-minute heart rate variations in association with apnoea and resumption of respiration, but the cyclical pattern was blunted. Sinus rate ranged from 65 to 90 per minute during sleep, in direct relationship to obstructed respiration and resumption of normal air exchange.

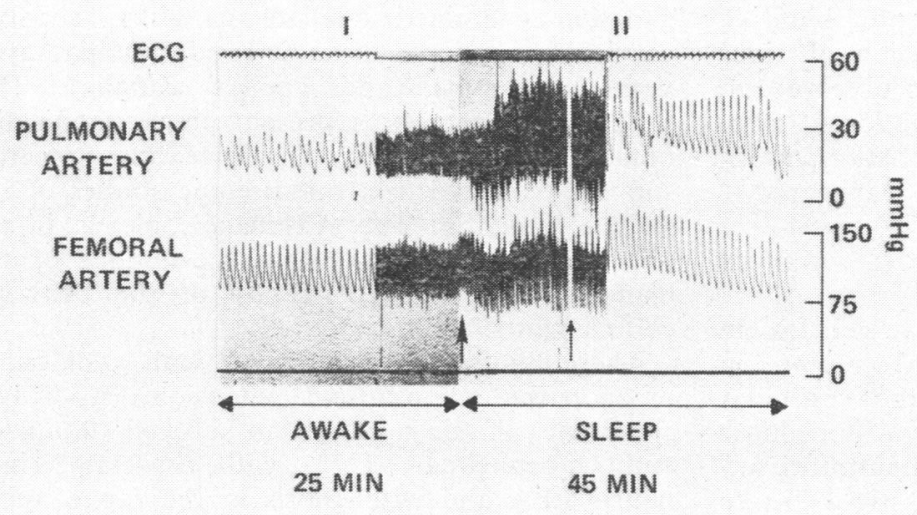

Fig. 2 Pulmonary $(P A)$ and femoral arterial $(F A)$ pressures recorded during 25 minutes of wakefulness and the following 45 minutes of sleep in patient 8. At the beginning and end of the graph (I and II respectively), paper speed was at $1 \mathrm{~mm} \mathrm{~s}^{-1}$ to allow analysis of the $P A$ and $F A$ components. Midgraph, paper speed was at $1 \mathrm{~mm} \mathrm{~min}^{-1}$. There is progressive increase of $P . A$ and $F A$ with repetitive sleep apnoea. First arrow indicates sleep onset, the second indicates flushing of the catheters. 


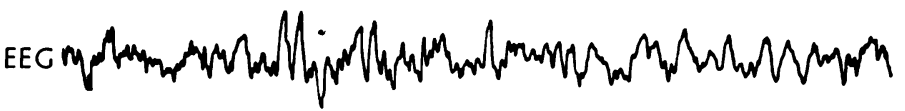

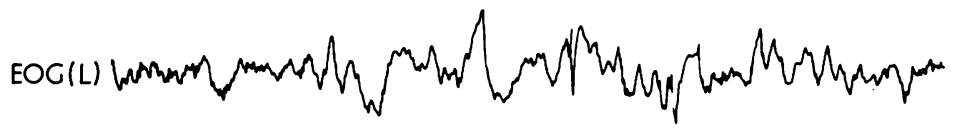

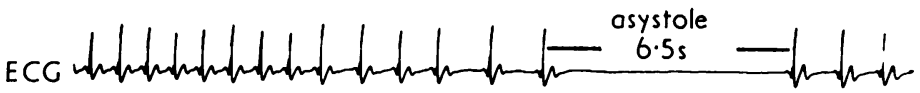

Fig. 36.5 seconds asystole during central sleep apnoea in patient 5 .

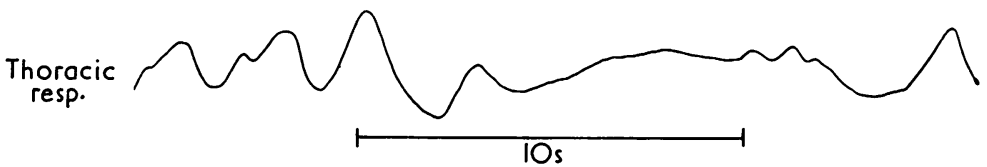

ACTION OF ATROPINE SULPHATE

The intravenous injection of atropine sulphate in patient 7 induced no change in respiration, apnoea, or sleep. There was a transient damping of systemic pressure variation which developed three minutes after injection and lasted for 20 minutes. Simultaneously, sinus tachycardia intervened with abolition of marked sinus arrhythmia. The five patients (cases 2, 3, 4, 6, and 9) who received oral atropine sulphate presented blunting of sinus bradycardia and accentuation of sinus tachycardia (Fig. 4). No effect was observed on sleep stages or respiration. As the action of atropine sulphate faded, the characteristic pattern associated with apnoea progressively reappeared.

SUMMARY OF THE NEUROPATHOLOGICAL FINDINGS IN CASE 11

There was a moderately severe nerve cell loss in the thoracolumbar intermediolateral column, in the dorsal motor vagus nucleus, the vestibular nuclei, and in the substantia nigra and locus caeruleus. No Lewy bodies or neurofibrillary tangles were seen in the substantia nigra. There was no striato-nigral or olivo-ponto-cerebellar degeneration. Marked gliosis was seen in the raphe nuclei throughout the brain stem and in the areas of nerve cell degeneration. There were no detectable neuronal losses in the region of the so-called pontine pneumotaxic centre and medullary centre. A mild peripheral neuropathy was present. The sympathetic ganglia showed marked juxta-neuronal vacuolation. This was observed in relation to a number of nerve cells in each ganglion examined (stellate ganglion, superior cervical ganglion, thoracic and lumbar sympathetic ganglia, and coeliac ganglia). This vacuolation seems to represent preganglionic terminal swellings; this interpretation receives support from electron microscopic studies of the stellate ganglion. The Auerbach's plexus in the proximal oesophagus and in the fundus of the bladder displayed hypertrophy of the Schwann cell elements.

These findings are consistent with a diagnosis of idiopathic orthostatic hypotension (Shy-Drager syndrome) of the multisystem subtype (Bannister and Oppenheimer, 1972) with associated mild neuropathy, and with gliosis in the raphe nuclei of the brain stem. 


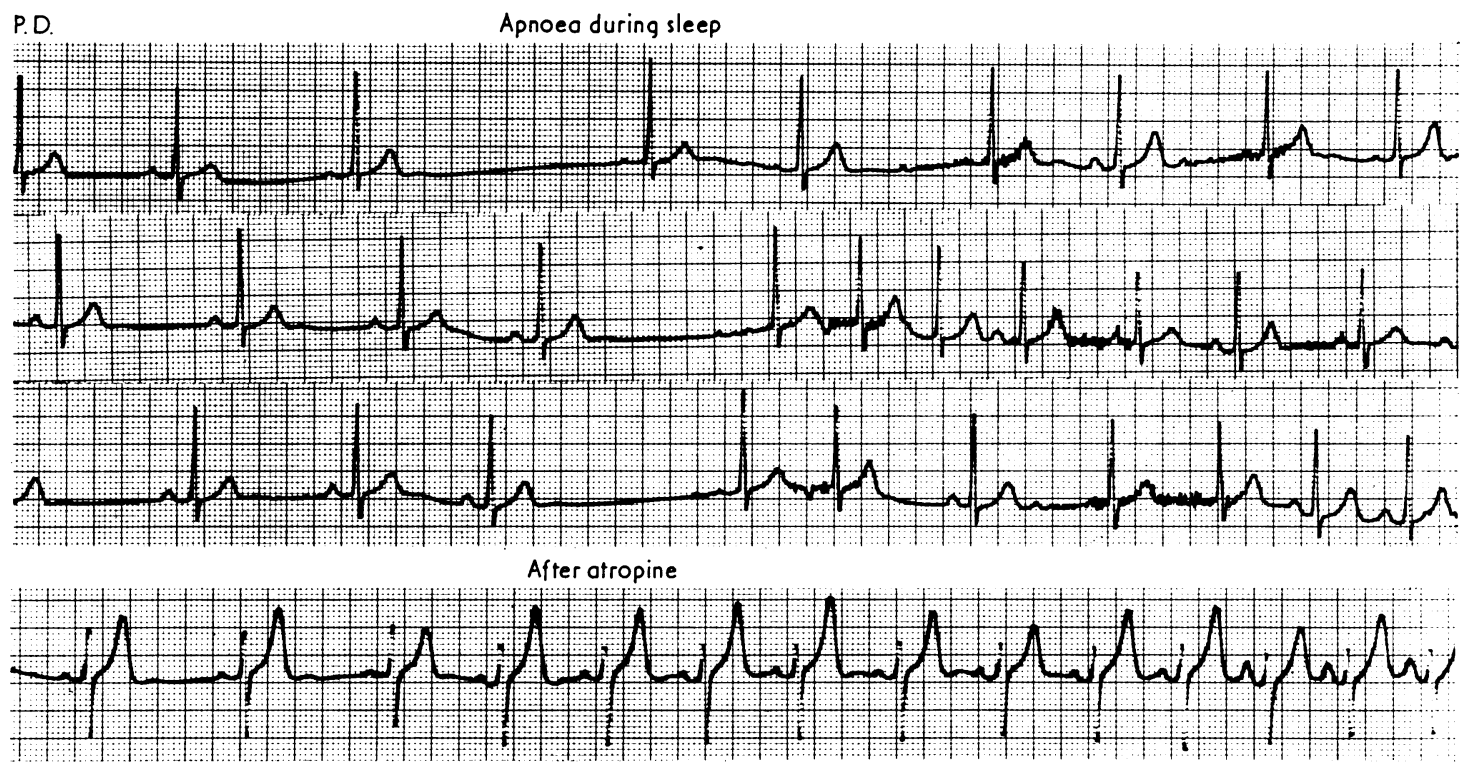

Fig. 4 Comparison of cardiac rhythm during sleep before atropine (sinus bradycardia) and after atropine in patient 4.

\section{Comments}

Awareness that the control of respiration is critically dependent on sleep states has developed progressively during recent years. Bolton and Herman (1974), Hathorn (1974), and Finer et al. (1976) have demonstrated increased respiratory rate and minute ventilation and increased variability of ventilation in REM compared to NREM sleep in infants. Orem et al. (1974) have reported changes in the activity of respiratory neurones during sleep in cats. Their findings imply a reduction in central respiratory drive during sleep with increased variability during REM sleep.

Analysis of our polygraphic data reveals that all patients have an abnormal respiratory pattern during sleep (sleep apnoea syndrome). This abnormal respiration markedly disturbs sleep and sleep stages, but REM sleep is only slightly reduced in most patients. The sleep related respiratory abnormality predominantly involves the upper airway, and a 'predominant upper airway sleep apnoea syndrome' may be characterised. Involvement of the pharyngeal muscles in the sleep induced obstruction has been postulated by numerous authors (see Guilleminault et al., 1976b for review) during the past 10 years. Recently, the possible involvement of the tongue in this obstructive process has been questioned. Sauerland and Harper (1976) demonstrated that during
NREM sleep in normals the genioglossal electromyogram is characterised by continuous discharge with substantially augmented activity during inspiration. When the subject enters REM sleep, however, the tonic genioglossal activity almost ceases except for small bursts during the inspiratory phase of respiration. These authors questioned if the loss of tonic genioglossal activity during REM sleep may not point to the cause of airway obstruction in patients with the sleep apnoea syndrome. Our polygraphic recordings demonstrate the complexity of the problem: the abnormal air exchange is sleep related; none of the patients have apnoea during wakefulness. In addition, the type of sleep-REM or NREMmay be instrumental in the presence or absence of respiratory alteration. A distinction must be made between 'total sleep dependent' patients, in whom the longest respiratory pauses very often are observed during REM sleep, and 'NREM sleep dependent' patients with entirely normal respiration during REM sleep. This latter group was clearly identified by normal blood gases measurements during REM sleep as well as by polygraphic monitoring.

The theoretical distinction between these two patient subgroups has practical haemodynamic implications. Cases 2 and 6 had normal haemodynamic measurements during REM sleep, and at the end of each sleep cycle (one NREM + REM 
sleep period), systemic and pulmonary arterial pressures had returned to normal values. In all other cases, systemic and pulmonary arterial pressures increased progressively from the beginning until the end of the total sleep period. The longterm effect of these haemodynamic changes on the right ventricle may be very different in the two subgroups. Resumption of normal breathing every 90 to 120 minutes (normal sleep cycle duration) with simultaneous disappearance of the hypoxia, hypercapnia, and mild acidosis during each REM cycle protects the patient from severe increases in pulmonary and systemic vascular pressures during sleep. This is not true in the 'total sleep dependent' patient group where pressures increase throughout the night, reaching dangerously high levels by morning.

We have little insight at present into the basic central mechanisms responsible for this difference. One might question if separate central nervous system imbalances are involved in each subgroup. We do have a better understanding about the neurogenic mechanisms involved in the development of haemodynamic anomalies and cardiac arrhythmias: when upper airway sleep apnoea was associated with generalised autonomic dysfunction (case 11), pulmonary arterial pressure values rose progressively during sleep in direct association with each apnoeic episode and related hypoxaemia and hypercapnia but without development of systemic hypertension, marked sinus arrhythmias, or extreme bradycardia. Similarly, when atropine sulphate (orally or intravenously) is given to patients, transitory blunting of sinus bradycardia and transient limitation of systemic pressure increase can be seen. It might be concluded that the progressive development of systemic hypertension during sleep in upper airway sleep apnoeic patients is mediated by the autonomic nervous system. If the known physiological action of the sympathetic and parasympathetic nerves is considered, it might also be suspected that a direct vagal inhibition is responsible for the lessening of the marked sinus arrhythmias recorded in association with upper airway sleep apnoea. Understanding of these phenomena is not purely academic: serious, lifethreatening arrhythmias and long asystoles are seen during sleep in this illness, and a better understanding of their mechanisms of appearance will contribute to better control.

Finally, a sleep apnoea syndrome may considerably reduce the life expectancy of patients with the Shy-Drager syndrome. Although this complication is not often suspected in these patients, it may not be uncommon.

\section{References}

Bannister, R., Ardill, L.. and Fentem, P. (1967). Defective autonomic control of blood vessels in idiopathic orthostatic hypotension. Brain, 90, 725746.

Bannister, R., and Oppenheimer. D. R. (1972). Degenerative diseases of the nervous system associated with autonomic failure. Brain, 95, 457-474.

Bolton, D. P. G., and Herman, S. (1974). Ventilation and sleep state in the newborn. Journal of Physiology (London), 240, 67-72.

Chekroverty, S., Barrocas, M., Barron, K. D., and Sharp, J. T. (1969). Hypoventilation syndrome and obesity: a polygraphic study. Transactions of the American Neurological Association, 94, 240-242.

Coccagna, G., Mantovani, M., Brignani, F., Parchi, C., and Lugaresi, E. (1972). Continuous recording of the pulmonary and systemic arterial pressure during sleep in syndromes of hypersomnia with periodic breathing. Bulletin de Physio-Pathologie Respiratoire, 8, 1159-1172.

Finer, N. N., Abrams, I. F., and Taeusch, H. W. Jr. (1976). Ventilation and sleep states in newborn infants. Journal of Pediatrics, 89, 142-147.

Guilleminault, C., Billiard, M., Montplaisir, J., and Dement, W. C. (1975a). Altered states of consciousness in disorders of daytime sleepiness. Journal of the Neurological Sciences. 26, 377-393.

Guilleminault, C., and Dement, W. C. (1977). 235 cases of excessive daytime sleepiness: diagnosis and tentative classification. Journal of the Neurological Sciences, 31, 13-27.

Guilleminault, C., Eldridge, F. L., Simmons, F. B., and Dement, W. C. (1975b). Sleep apnea syndrome: can it induce hemodynamic changes? Western Journal of Medicine, 123, 7-16.

Guilleminault. C., Eldridge, F., Simmons, F. B., and Dement, W. C. (1976a). Sleep apnea in eight children. Pediatrics, 58, 23-30.

Guilleminault, C., Tilkian, A., and Dement. W. C. (1976b). The sleep apnea syndromes. Annual $R e$ view of Medicine, 27, 465-484.

Guilleminault, C., Tilkian, A., Eldridge, F. L., Simmons, F. B., and Dement, W. C. (1977). Sleep apnea syndrome due to upper airway obstruction: a review of 25 cases. Archives of Internal Medicine, 137, 296-300.

Hathorn, M. K. S. (1974). The state and depth of breathing in newborn infants in different sleep states. Journal of Physiology (London), 243, 101107.

MacGregor, M. I., Block. A. J., and Ball. W. C. (1970). Serious complications and sudden death in the Pickwickian syndrome. Johns Hopkins Medical Journal, 126, 279-295.

Metropolitan Life Insurance Company (1959). Statistical Bulletin, 40, November-December.

Orem, J., Montplaisir, J.. and Dement, W. C. (1974). Changes in the activity of respiratory neurons during sleep. Brain Research, 82, 309-315.

Robinson, R. R. (ed.) (1974). SIDS 1974. The 
Canadian Foundation for the Study of Infant Death, Toronto, Canada.

Sackner, M. A., Landa, J., Forrest, F., and Greeneltch, D. (1974). Periodic sleep apnea: chronic sleep deprivation related to intermittent upper airway obstruction and central nervous system disturbance. Chest, 67, 164-171.

Sauerland, E. K., and Harper, R. M. (1976). The human tongue during sleep: electromyographic activity of the genioglossus muscle. Experimental Neurology, 51, 160-170.

Tassinari, C. A., Dalla Bernardina, B., Cirignotta, F., and Ambrosetto, G. (1972). Apnetic periods and the respiratory related arousal patterns during sleep in the Pickwickian syndrome: a polygraphic study. Bulletin de Physio-Pathologie Respiratoire, 8, 1087-1102.

Tilkian, A. G., Guilleminault, C., Schroeder, J. S., Lehrman, K. L., Simmons, F. B., and Dement, W. C. (1976). Sleep induced apnea syndrome: hemodynamic studies during wakefulness and sleep. Annals of Internal Medicine, 85, 714-719.

Williams, R., Karacan, I., and Hursch, C. (1947). Electroencephalography (EEG) of Human Sleep: Clinical Applications. John Wiley and Sons: New York. 